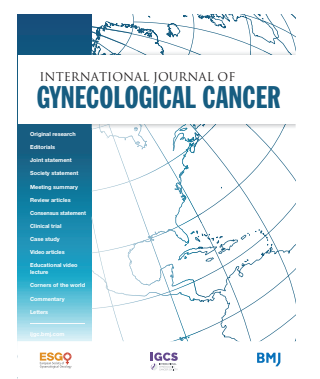

\title{
SUCCOR study: an international European cohort observational study comparing minimally invasive surgery versus open abdominal radical hysterectomy in patients with stage IB1 cervical cancer
}

Luis Chiva (1) , ${ }^{1}$ Vanna Zanagnolo, ${ }^{2}$ Denis Querleu, ${ }^{3}$ Nerea Martin-Calvo, ${ }^{4}$ Juan Arévalo-Serrano, ${ }^{5}$ Mihai Emil Căpîlna, ${ }^{6}$ Anna Fagotti, ${ }^{7}$ Ali Kucukmetin, ${ }^{8}$ Constantijne Mom, ${ }^{9}$ Galina Chakalova, ${ }^{10}$ Shamistan Aliyev, ${ }^{11}$ Mario Malzoni, ${ }^{12}$ Fabrice Narducci (D) ${ }^{13}$ Octavio Arencibia,,${ }^{14}$ Francesco Raspagliesi, ${ }^{15}$ Tayfun Toptas, ${ }^{16}$ David Cibula, ${ }^{17}$ Dilyara Kaidarova, ${ }^{18}$ Mehmet Mutlu Meydanli (D), ${ }^{19}$ Mariana Tavares, ${ }^{20}$ Dmytro Golub, ${ }^{21}$ Anna Myriam Perrone (D) ,22 Robert Poka, ${ }^{23}$ Dimitrios Tsolakidis, ${ }^{24}$ Goran Vujić, ${ }^{25}$ Marcin A Jedryka (D) ${ }^{26}$ Petra L M Zusterzeel, ${ }^{27}$ Jogchum Jan Beltman, ${ }^{28}$ Frederic Goffin, ${ }^{29}$ Dimitrios Haidopoulos, ${ }^{30}$ Herman Haller, ${ }^{31}$ Robert Jach, ${ }^{32}$ Iryna Yezhova, ${ }^{33}$ Igor Berlev, ${ }^{34}$ Margarida Bernardino, ${ }^{35}$ Rasiah Bharathan, ${ }^{36}$ Maximilian Lanner, ${ }^{37}$ Minna M Maenpaa, ${ }^{38}$ Vladyslav Sukhin (D) , ${ }^{39}$ Jean-Guillaume Feron, ${ }^{40}$ Robert Fruscio, ${ }^{41,42}$ Kersti Kukk, ${ }^{43}$ Jordi Ponce, ${ }^{44}$ Jose Angel Minguez, ${ }^{45}$ Daniel Vázquez-Vicente (I) ${ }^{45}$ Teresa Castellanos, ${ }^{45}$ Enrique Chacon, ${ }^{46}$ Juan Luis Alcazar (D) , ${ }^{47}$ et al,, On behalf of the SUCCOR study Group

- Additional material is published online only. To view please visit the journal online (http://dx.doi.org/10.1136/ijgc2020-001506).

For numbered affiliations see end of article.

Correspondence to Dr Luis Chiva, Obstetrics and Gynecology, Clinica Universidad de Navarra, 28027 Madrid, Spain; Ichiva@unav.es

For 'Presented at statement' see end of article.

Received 20 April 2020 Revised 29 June 2020 Accepted 1 July 2020 Published Online First 11 August 2020

Check for updates

(C) IGCS and ESGO 2020. № commercial re-use. See rights and permissions. Published by BMJ.

To cite: Chiva L, Zanagnolo V, Querleu D, et al. Int J Gynecol Cancer 2020;30:1269-1277.

\section{HIGHLIGHTS}

- Minimally invasive surgery in patients with FIG0 2009 stage IB1 cervical cancer increased the risk of relapse and death.

- Patients that underwent minimally invasive surgery using a uterine manipulator had a 2.76-times higher hazard of relapse.

- Avoiding uterine manipulator and implementing a protective vaginal closure was associated with disease-free-survival similar to that of open surgery.

\section{ABSTRACT}

Background Minimally invasive surgery in cervical cancer has demonstrated in recent publications worse outcomes than open surgery. The primary objective of the SUCCOR study, a European, multicenter, retrospective, observational cohort study was to evaluate disease-free survival in patients with stage IB1 (FIGO 2009) cervical cancer undergoing open vs minimally invasive radical hysterectomy. As a secondary objective, we aimed to investigate the association between protective surgical maneuvers and the risk of relapse.

Methods We obtained data from 1272 patients that underwent a radical hysterectomy by open or minimally invasive surgery for stage IB1 cervical cancer (FIG0 2009) from January 2013 to December 2014. After applying all the inclusion-exclusion criteria, we used an inverse probability weighting to construct a weighted cohort of 693 patients to compare outcomes (minimally invasive surgery vs open). The first endpoint compared disease-free survival at 4.5 years in both groups. Secondary endpoints compared overall survival among groups and the impact of the use of a uterine manipulator and protective closure of the colpotomy over the tumor in the minimally invasive surgery group.

Results Mean age was 48.3 years (range; $23-83$ ) while the mean BMI was $25.7 \mathrm{~kg} / \mathrm{m}^{2}$ (range; $15-49$ ). The risk of recurrence for patients who underwent minimally invasive surgery was twice as high as that in the open surgery group (HR, 2.07; 95\% Cl, 1.35 to 3.15; $\mathrm{P}=0.001$ ). Similarly, the risk of death was 2.42-times higher than in the open surgery group ( $\mathrm{HR}, 2.45 ; 95 \% \mathrm{Cl}, 1.30$ to $4.60, \mathrm{P}=0.005)$. Patients that underwent minimally invasive surgery using a uterine manipulator had a 2.76-times higher hazard of relapse (HR, 2.76; $95 \% \mathrm{Cl}, 1.75$ to $4.33 ; \mathrm{P}<0.001)$ and those without the use of a uterine manipulator had similar disease-free-survival to the open surgery group (HR, 1.58; $95 \% \mathrm{Cl}, 0.79$ to $3.15 ; \mathrm{P}=0.20$ ). Moreover, patients that underwent minimally invasive surgery with protective vaginal closure had similar rates of relapse to those who underwent open surgery $(\mathrm{HR}, 0.63 ; 95 \% \mathrm{Cl}, 0.15$ to 2.59 ; $\mathrm{P}<0.52$ ).

Conclusions Minimally invasive surgery in cervical cancer increased the risk of relapse and death compared with open surgery. In this study, avoiding the uterine manipulator and using maneuvers to avoid tumor spread at the time of colpotomy in minimally invasive surgery was associated with similar outcomes to open surgery. Further prospective studies are warranted.

\section{INTRODUCTION}

On November 16, 1898, in Vienna, Dr. Ernst Wertheim who was 34 years' old at the time - performed the first radical abdominal hysterectomy for cervical cancer in 


\section{Original research}

Europe. ${ }^{1}$ Since then, early cervical cancer has been successfully treated by radical hysterectomy over the past 120 years. Initially, by open or vaginal approach, and more recently (since 1991), by minimally invasive surgery, ${ }^{2} 3$ either by laparoscopy or robotic surgery. For 25 years, multiple retrospective publications reported the feasibility, advantages, and oncologic safety of this minimally invasive approach. ${ }^{4-7}$ However, two recent manuscripts published in October of 2018 in NEJM (the LACC trial and a large epidemiologic study involving women from Cancer-Accredited hospitals in the United States) ${ }^{8}{ }^{9}$ demonstrated higher rates of recurrence and death in patients that underwent minimally invasive radical hysterectomy. Subsequently, a number of retrospective studies corroborated these findings, ${ }^{10-15}$ resulting in a recent modification of the NCCN, European Society of Gynecological Oncology (ESGO), and ESMO guidelines. ${ }^{16-18}$

The unexpected results of the LACC trial and other retrospective studies led to an ongoing discussion regarding the ideal surgical approach in patients with early cervical cancer. Also, the potential of implementing new trials containing a minimally invasive approach arm has been difficult, given the challenges of offering an option of treatment that has been deemed oncologically inferior. Moreover, the reasons for the inferior outcomes of minimally invasive surgery have not yet been elucidated. ${ }^{19}$

In 2019, the ESGO conducted a survey named "after LACC trial", attempting to understand the perspective of their members on this topic. The study showed that $83 \%$ of respondents did not expect these results, and 4 months after the publication of the LACC trial, $56 \%$ had changed their surgical approach from minimally invasive surgery to open radical hysterectomy. ${ }^{20}$ In Europe, there was a lack of updated information regarding the outcomes on the surgical approach of radical hysterectomy. With this in mind, we designed the SUCCOR (Surgery in Cervical Cancer, Observational, Retrospective) study, comparing minimally invasive vs open abdominal radical hysterectomy in patients with FIG0 2009 stage IB1 cervical cancer. The goal of this study was to determine disease-free survival of radical hysterectomy between the two surgical approaches in Europe. Second, we aimed to explore the risk of relapse between the two approaches, with a particular focus on the surgical maneuvers (use of uterine manipulator, protective closure of colpotomy over the tumor). Lastly, we also wished to analyze the influence of the aforementioned variables based on tumor size.

\section{METHODS}

\section{Study design and endpoints}

The study was a European, multicenter, observational, retrospective, cohort study spanning from January 1, 2013 to December 31,2014 with the primary goal of comparing disease-free survival at 4.5 years in patients who underwent a laparoscopic or robotic radical hysterectomy vs abdominal hysterectomy for FIGO 2009 stage IB1 cervical cancer. The secondary endpoints included a comparison of overall survival at 4.5 years between groups and to explore the association between specific surgical maneuvers and the risk of relapse. More specifically, we aimed to determine if the use of a uterine manipulator and protective vaginal closure over the cervix to avoid tumor spread at the time of the colpotomy might impact the outcome of patients undergoing minimally invasive surgery. Lastly, we investigated the influence of tumor diameter on oncologic outcomes. Disease-free survival was defined as the time from the date of the radical hysterectomy to time of relapse or last contact. Overall survival was calculated from the date of the radical hysterectomy to the time of last contact or death from cervical cancer. We inquired on the use of the uterine manipulator given previously published data suggesting its use as an etiology for the worse outcomes in minimally invasive surgery. ${ }^{21} 22$ The protective vaginal closure over the tumor has been advocated to avoid contact between the cervical tumor and the abdominal cavity after the colpotomy. ${ }^{23}$

We registered the study at ClinicalTrials.gov with the Identifier NCT03958305. ${ }^{24} \mathrm{~A}$ copy of the protocol is provided in the online supplementary appendix. All authors contributed meaningfully to the conception or design of the work or the acquisition, analysis, or interpretation of data for the study. The authors confirm the completeness and accuracy of the data and analyzes, the fidelity of the study to the protocol, and the final approval of the version to be published.

\section{Inclusion and exclusion criteria}

We designed a list of inclusion-exclusion criteria to define a homogeneous population. Patients were eligible if they underwent a radical hysterectomy in a European Institution for stage IB1 cervical cancer (FIG0 2009), from January 1, 2013 to December 31, 2014. Inclusion criteria included patients age 18 years' old or older and squamous cell carcinoma, adenocarcinoma, or adenosquamous carcinoma. A pelvic MRI confirming a tumor diameter less or equal than $4 \mathrm{~cm}$ with no parametrial invasion and a preoperative CT scan, $\mathrm{MRI}$, or PET-CT without extra-cervical metastatic disease, were mandatory. The operative report had to describe either a Type B-C radical hysterectomy by minimally invasive surgery (laparoscopic or robotic) or by open surgery with a bilateral pelvic lymphadenectomy, including at least a total of 10 pelvic nodes. Those who only underwent sentinel lymph node mapping were allowed in the study. There had to be documentation of tumor size, margins, and nodal status.

Patients were excluded when any other histological type was diagnosed; if tumor size was larger than $4 \mathrm{~cm}$; and when there was a past history of any invasive tumor, previous chemotherapy or radiation, suspicious lymph nodes, or metastatic disease on preoperative imaging. Conversion from minimally invasive surgery to laparotomy was cause for exclusion. Finally, patients with cervical conization prior to radical hysterectomy were excluded from the primary endpoint analysis. Reasons for not including patients with the previous conization were as follows: cone biopsies were often performed at outside institutions thus leading to missing critical pathological information; insufficient pathology report to stage the tumor adequately; lack of consensus on measurement of tumor size when cone biopsy and final specimen both had residual disease; consideration that conization might be a source of bias given that, if no residual tumor, then risk for tumor dissemination may be lower than that of patients with gross tumor; and lastly, we are currently working on an additional manuscript that focuses on this patient group within the SUCCOR database.

\section{Accrual and data source}

We invited all ESGO members to participate in this study. Researchers from 126 institutions in 29 European countries registered and 
contributed to the project. After obtaining ethical consent from our central IRB, we required a Certificate of Approval or a Letter of Exemption by the local Ethical Committees from all the investigators. An anonymized complete Case Record Form, including 123 items (online supplementary file 1), was sent to all the investigators. After completing the case collection, all researchers signed a final declaration affirming that all the submitted data matched the data in the patients' charts. As far as the researcher was able to analyze, data included all cases operated on at the respective institutions.

\section{Statistical analysis}

In our primary analyzes when comparing open vs minimally invasive surgical approach, we hypothesized $10 \%$ of relapse in the group that underwent open surgery. Assuming a two-sided alfa error of $5 \%$ and an $80 \%$ of statistical power, 261 women were needed in each group to detect a difference between groups of $9 \%$ in the risk of relapse. We used inverse probability of treatment weighting based on propensity score to construct a weighted cohort of patients who differed with respect to surgical approach but were similar with respect to other measured characteristics. ${ }^{25} \mathrm{To}$ calculate the inverse probability of treatment weights, each patient's propensity to undergo open hysterectomy was estimated using a logistic regression model that included predictor variables that had been selected based on their a priori possibility of confounding the relationship between surgical approach and survival (largest tumor diameter, tumor grade, lymphovascular invasion, depth of invasion, margins status, nodal status, and adjuvant therapy) ${ }^{26}$ We assigned patients who underwent open surgery a weight of $1 /$ (propensity score) and those who underwent minimally invasive surgery a weight of $1 /$ ( 1 - propensity score). To reduce the variability in the inverse probability of treatment-weighted models, we used stabilized weights in all the analyzes. To explore whether the observed associations differed according to largest tumor size, we performed a subgroup analysis.

In the secondary analyzes, we calculated the inverse probability of treatment weights considering open surgery, minimally invasive surgery without uterine manipulator, and minimally invasive surgery with a uterine manipulator. We used a multinomial logistic regression model with the same predictors in the primary analysis. We obtained three propensity scores and assigned each patient weight of $1 /$ (the correspondent propensity score). Finally, we followed the same calculations considering open surgery and minimally invasive surgery with and without vaginal protective closure. We applied the same multinomial logistic regression model with the identical predictors in the principal analysis. Besides, we obtained three propensity scores as in the evaluation of the uterine manipulator use.

Participants with missing values in quantitative variables were excluded from the analyzes. Missing values in qualitative variables were classified into a new category. Distributions of categorical variables were compared using the chi-square test in the unweighted cohort and weighted logistic-regression models in the weighted cohort. Quantitative variables were compared using the student $t$-test in the unweighted cohort and weighted linearregression models in the weighted cohort.

We compared disease-free survival and overall survival using the inverse probability of treatment-weighted log-rank test and plotted weighted incidence functions using Nelson-Aalen survival

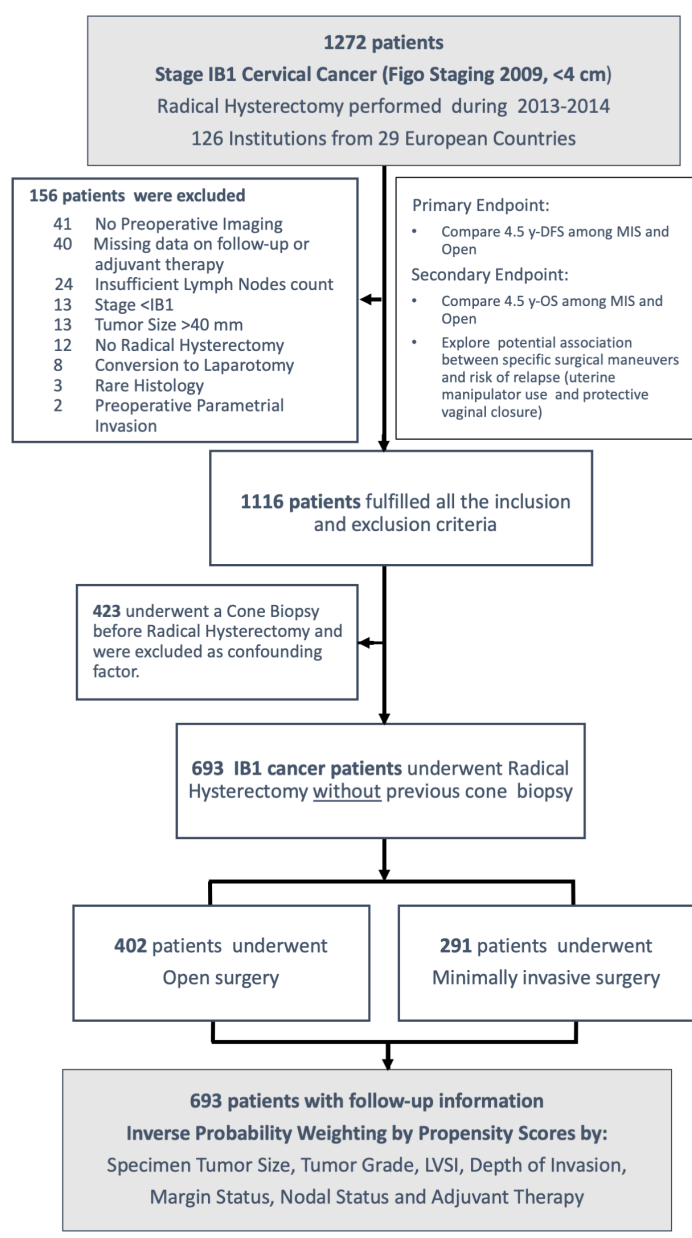

Figure 1 Flowchart of study population.

curves. We estimated the hazard ratio (and 95\% Cl) for diseasefree survival and overall survival after each of the different surgical approach using open surgery as the category of reference. After analyzing the proportionality of hazard with the specific test and assessing surgical approach was not a time varying covariate, we used weighted Cox proportional-hazards models in all the analyzes. The analyzes were performed with Stata 15.0. and SPSS v26.0. P-values below 0.05 were considered statistically significant.

\section{RESULTS}

\section{Study population}

From May 15 to November 15, 2019, we received data from a total of 1272 patients that underwent a radical hysterectomy in Europe for stage IB1 cervical cancer during 2013-2014 (Figure 1). A total of 156 patients did not meet the inclusion-exclusion criteria or had missing information on follow-up and were excluded: of the remaining 1116 patients, $37.9 \%$ (423 patients) had undergone a cone biopsy previous to radical hysterectomy and were excluded from the analysis. Data on 693 patients was analyzed for the primary endpoint. Of these patients, 291 underwent minimally invasive and 402 an open radical hysterectomy (see online supplementary appendix). Among patients in the minimally invasive surgery group, 228 (78.5\%) underwent laparoscopic surgery and $63(21.5 \%)$ robotics surgery. Patients in the minimally invasive surgery group had more favorable prognostic features. They 
Table 1 Selected characteristics of patients that underwent radical hysterectomy for stage IB1 cervical carcinoma, by surgical approach, before and after inverse probability of treatment weighting

\begin{tabular}{|c|c|c|c|c|c|c|}
\hline \multirow[b]{2}{*}{ Baseline characteristics } & \multicolumn{2}{|c|}{$\begin{array}{l}\text { Before inverse probability } \\
\text { weighting }\end{array}$} & \multirow[b]{2}{*}{ P-value } & \multicolumn{3}{|c|}{ After inverse probability weighting } \\
\hline & $\begin{array}{l}\text { Open surgery } \\
(n=402)\end{array}$ & $\begin{array}{l}\text { Minimally inv. } \\
\text { surgery } \\
(n=291)\end{array}$ & & $\begin{array}{l}\text { Open surgery } \\
(n=402)\end{array}$ & $\begin{array}{l}\text { Minimally inv. } \\
\text { surgery } \\
(n=291)\end{array}$ & P-value \\
\hline Age-yr & $48.50(10.60)$ & $47.93(11.51)$ & 0.51 & $48.31(10.75)$ & $48.10(11.26)$ & 0.80 \\
\hline Body-mass index $-\mathrm{kg} / \mathrm{m}_{2}$ & $25.98(4.73)$ & $25.49(5.62)$ & 0.27 & $25.92(5.51)$ & $25.92(4.81)$ & 0.48 \\
\hline PS 0 & $351(87.31)$ & $256(87.97)$ & & $353(87.81)$ & 257 (88.31) & \\
\hline PS1 & $41(10.19)$ & $20(6.87)$ & & $37(9.20)$ & $20(6.87)$ & \\
\hline Not reported & $10(2.48)$ & $15(5.15)$ & & $12(3.89)$ & $14(4.81)$ & \\
\hline Preoperative clinical size (\%) & & & $<0.001$ & & & 0.058 \\
\hline Not evaluable & $8(1.99)$ & $11(3.78)$ & & $8(1.95)$ & $12(4.12)$ & \\
\hline \multicolumn{7}{|c|}{ Radical histerectomy pathology report } \\
\hline $\begin{array}{l}\text { Largest diameter in path- } \\
\text { report }(\mathrm{mm})\end{array}$ & 24.07 (10.13) & $22.57(9.46)$ & 0.05 & 23.66 (9.99) & $23.15(9.60)$ & 0.501 \\
\hline Largest tumor diameter (\%) & & & 0.270 & & & 0.941 \\
\hline$\leq 2 \mathrm{~cm}$ & $160(39.80)$ & $128(43.99)$ & & $168(41.63)$ & $120(41.35)$ & \\
\hline$>2 \mathrm{~cm}$ & $242(60.20)$ & $163(56.01)$ & & $235(58.37)$ & $170(58.65)$ & \\
\hline Final histological grade (\%) & & & 0.266 & & & 0.983 \\
\hline Grade 1 & $59(14.68)$ & $42(14.43)$ & & $59(14.65)$ & $43(14.71)$ & \\
\hline Grade 2 & $197(49.0)$ & $130(44.67)$ & & $188(46.66)$ & $135(46.54)$ & \\
\hline Not reported & $53(13.18)$ & $21(7.22)$ & & $43(10.80)$ & $32(11.06)$ & \\
\hline Depth of Invasion (\%) & & & 0.069 & & & 0.998 \\
\hline Superficial $<1 / 3$ & $77(19.15)$ & 65 (22.34) & & $81(20.23)$ & $59(20.24)$ & \\
\hline Intermediate $>1 / 3$ and $<1 / 3$ & 135 (33.58) & $75(25.77)$ & & $121(30.11)$ & $87(29.86)$ & \\
\hline Deep $>2 / 3$ & $131(32.59)$ & $68(23.37)$ & & $116(28.77)$ & $85(29.31)$ & \\
\hline Not reported & $59(14.68)$ & $83(28.52)$ & & $84(20.89)$ & $59(20.59)$ & \\
\hline Margins status (\%) & & & 0.151 & & & 0.954 \\
\hline Negative & 357 (88.81) & $268(92.10)$ & & $364(90.13)$ & $262(90.13)$ & \\
\hline Positive or close $<2 \mathrm{~mm}$ & $45(11.19)$ & $23(7.90)$ & & $39(9.74)$ & $29(9.87)$ & \\
\hline Lymph node status (\%) & & & 0.106 & & & 0.916 \\
\hline Negative & $336(83.58)$ & $256(87.97)$ & & $345(85.74)$ & $248(85.45)$ & \\
\hline Positive & 66 (16.42) & $35(12.03)$ & & $57(14.26)$ & $43(14.55)$ & \\
\hline FIGO staging $2018(\%)$ & & & 0.015 & & & 0.129 \\
\hline IB1 & $118(29.35)$ & $116(39.86)$ & & $126(31.18)$ & $107(36.71)$ & \\
\hline IB2 & 207 (51.49) & $129(44.33)$ & & $210(52.13)$ & $129(44.28)$ & \\
\hline $\mathrm{II}-\mathrm{II}<4 \mathrm{~cm}$ & 77 (19.15) & $46(15.81)$ & & 67 (16.69) & $55(19.01)$ & \\
\hline
\end{tabular}


Table 1 Continued

\begin{tabular}{|c|c|c|c|c|c|c|}
\hline \multirow[b]{2}{*}{ Baseline characteristics } & \multicolumn{2}{|c|}{$\begin{array}{l}\text { Before inverse probability } \\
\text { weighting }\end{array}$} & \multirow[b]{2}{*}{ P-value } & \multicolumn{3}{|c|}{ After inverse probability weighting } \\
\hline & $\begin{array}{l}\text { Open surgery } \\
(\mathrm{n}=402)\end{array}$ & $\begin{array}{l}\text { Minimally inv. } \\
\text { surgery } \\
(n=291)\end{array}$ & & $\begin{array}{l}\text { Open surgery } \\
(n=402)\end{array}$ & $\begin{array}{l}\text { Minimally inv. } \\
\text { surgery } \\
(n=291)\end{array}$ & P-value \\
\hline Post-adjuvant therapy (\%) & & & 0.002 & & & 0.776 \\
\hline None & $166(41.29)$ & $154(52.92)$ & & $191(47.31)$ & $140(48.40)$ & \\
\hline
\end{tabular}

Counts in the weighted cohort may not sum to expected totals owing to rounding. Percentages may not total 100 because of rounding, and disagreements between numbers and percentages in the weighted cohort are the result of rounding of noninteger number values. Distributions of categorical variables were compared using the chi-square test in the unweighted cohort and weighted logisticregression models in the weighted cohort. Quantitative variables were compared using the student $t$-test in the unweighted cohort and weighted linear-regression models in the weighted cohort.

were more likely to have smaller tumors, a lower percentage of positive nodes, and less frequently received adjuvant therapy. The final cohort was composed of 693 patients (open surgery, $n=402$; minimally invasive surgery group $n=291$ ) and, after weighting, did not show statistical differences between groups in the selected covariates (Table 1).

\section{Survival analysis based on surgical approach}

Patients in the weighted cohort had a median follow-up of 59 months (range; 1-83), 56 months in the minimally invasive surgery group, and 60 months in the open group. At 4.5 years of follow-up, there were $60(20.6 \%)$ relapses in 291 patients in the minimally invasive surgery group while in the open surgery group there were 47 (11.7\%) relapses in 402 patients. Disease-free survival at 4.5 years was $79 \%$ in the minimally invasive group and $89 \%$ in the open surgery group $(P=0.0003)$. The risk of recurrence for patients who underwent minimally invasive surgery was twice that of the open surgery group ( $\mathrm{HR}$, $2.07 ; 95 \% \mathrm{Cl}, 1.35$ to $3.15 ; \mathrm{P}=0.001$ ) (Figure $2 \mathrm{~A}$ ). We confirmed those differences in the subgroup of patients with tumors greater than $2 \mathrm{~cm}(\mathrm{HR}, 2.31 ; ; 95 \% \mathrm{Cl}, 1.37$ to $3.90 ; \mathrm{P}=0.002)$ while in the group of patients with tumors smaller or equal than $2 \mathrm{~cm}$ those differences were not significant (HR, 1.63; 95\% $\mathrm{Cl}, 0.79$ to $3.40 ; \mathrm{P}=0.19$ )

Minimally invasive surgery was also associated with a lower rate of overall survival than open surgery. A total of 28 patients died in the minimally invasive surgery group while 21 patients died in the open surgery group. The risk of death was 2.42-times higher than in the open surgery group ( $\mathrm{HR}, 2.42 ; 95 \% \mathrm{Cl}, 1.34$ to $4.39 ; \mathrm{P}=0.004)$. At 4.5 years, the proportion of living patients was $89 \%$ in the minimally invasive and $97 \%$ in the open surgery group (Figure 2B). Again, these differences were significant in patients with tumors greater than $2 \mathrm{~cm}(\mathrm{HR}, 2.26 ; 95 \% \mathrm{Cl}, 1.18$ to 4.36; $\mathrm{P}=0.014)$ but not in patients with tumors smaller or equal to $2 \mathrm{~cm}(\mathrm{HR}, 2.77 ; 95 \% \mathrm{Cl}, 0.91$ to 8.47 ; $\mathrm{P}=0.072$ )

\section{Impact of uterine manipulator in minimally invasive surgery}

A weighted cohort was built with the same covariates used in the surgical approach analysis, 652 patients (open, $n=402$; minimally invasive surgery without manipulator, $n=106$; and minimally invasive surgery with the manipulator, $n=144)$. After the inverse probability of treatment weighting, survival analysis demonstrated a negative impact of uterine manipulators on disease-free survival. The manipulator group experienced $38(26.3 \%)$ relapses in 144 patients compared with $17(16 \%)$ relapses in 106 patients without a manipulator. The disease-free survival at 4.5 years was $73 \%$ in the uterine manipulator group and $83 \%$ in those without $(\mathrm{P}=0.0001)$. The patients that underwent minimally invasive surgery with a uterine manipulator had a 2.76-times higher chance of a relapse compared with those in the open approach (HR, 2.76; 95\% Cl, 1.75 to 4.33; $\mathrm{P}<0.001$ ) (Figure 3A). The adverse effect of the uterine manipulator significantly impacted patients with tumors greater than $2 \mathrm{~cm}(\mathrm{HR}, 3.05 ; 95 \% \mathrm{Cl}, 1.73$ to 5.38; $\mathrm{P}<0.001$ ) while tumors less or equal than $2 \mathrm{~cm}$ did not show differences (HR, 2.25; $95 \% \mathrm{Cl}, 0.96$ to $5.26 ; \mathrm{P}=0.06)$. Patients that
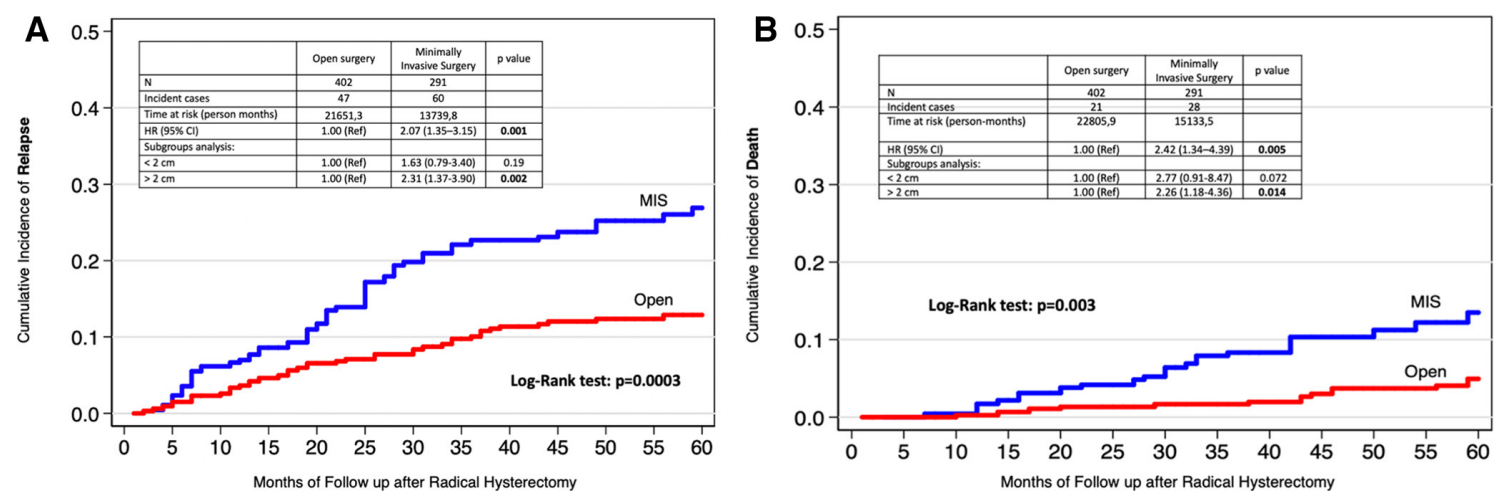

Figure 2 (A) Hazard ratios and 95\% confidence intervals for the risk of relapse by type of surgical approach. Adjusted cohort using inverse probability weighting by propensity scores. (B) Hazard ratios and $95 \%$ confidence intervals for the overall survival by type of surgical approach. Adjusted using inverse probability weighting by propensity scores. 


\section{Original research}
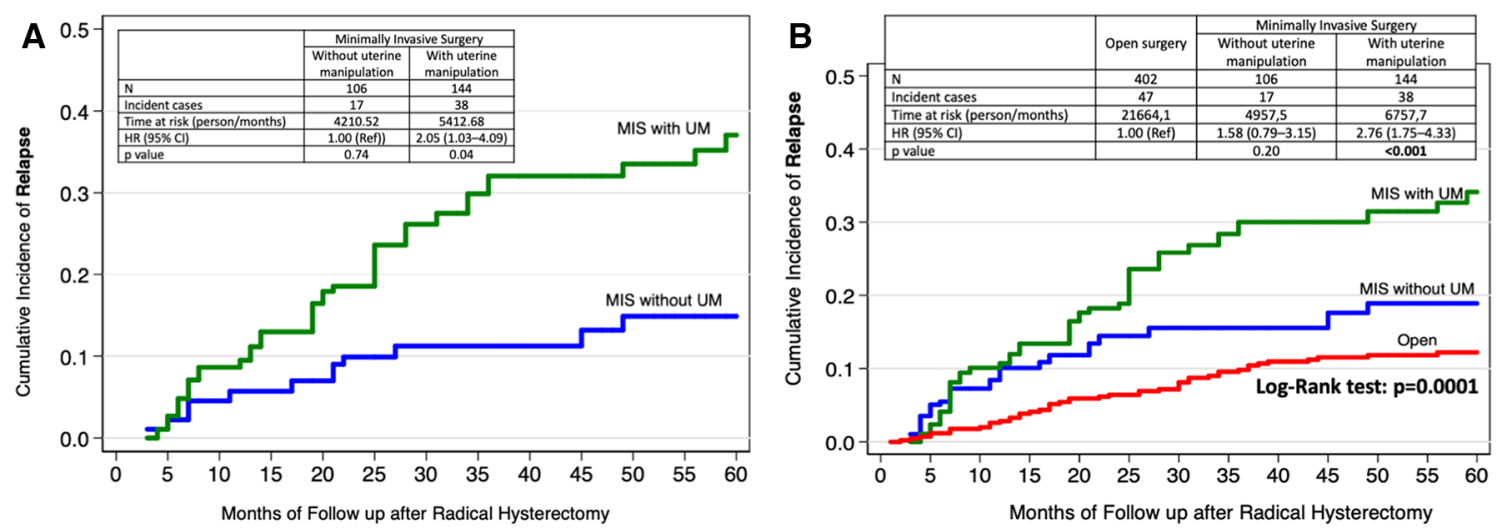

Figure 3 (A) Hazard ratios and 95\% confidence intervals for the risk of relapse by type of intervention in patients that underwent minimally invasive surgery (use of uterine manipulator vs not used). Adjusted using inverse probability weighting by propensity scores. (B) Hazard ratios and $95 \%$ confidence intervals for the risk of relapse by type of intervention in patients that underwent minimally invasive surgery (use of uterine manipulator vs no use) compared with patients with open radical hysterectomy Adjusted using inverse probability weighting by propensity scores.

underwent minimally invasive surgery without the uterine manipulator had similar rates of relapse to those who underwent open surgery (HR, $1.58 ; 95 \% \mathrm{Cl}, 0.79$ to $3.15 ; \mathrm{P}=0.20$ ). The use of a uterine manipulator adversely impacted overall survival in patients who underwent minimally invasive surgery (HR, 3.00; $95 \% \mathrm{Cl}, 1.60$ to $5.63 ; \mathrm{P}=0.001$ ). In this case, the overall survival was significantly lower in all tumor sizes (tumors greater than $2 \mathrm{~cm}(\mathrm{HR}, 2.69 ; 95 \% \mathrm{Cl}, 1.22$ to $5.89 ; \mathrm{P}=0.013$ ) and tumors smaller or equal to $2 \mathrm{~cm}(\mathrm{HR}, 3.84 ; 95 \% \mathrm{Cl}, 1.11$ to 13.26 ; $\mathrm{P}=0.033$ ) (Figure 3B) (See online supplementary file 1).

\section{Protective vaginal closure in minimally invasive surgery}

Lastly, we constructed a new weighted cohort to explore the impact of protective vaginal closure (Figure 4A). We used the same covariates used before (largest tumor diameter, tumor grade, lymphovascular invasion, depth of invasion, margins status, nodal status, and adjuvant therapy) to balance the three groups of patients: minimally invasive surgery patients with and without protective vaginal closure, and those with open surgery. Within this weighted cohort, 52 (25\%) of 207 patients had a relapse among those without protective vaginal closure, while only $3(7 \%)$ of 43 recurred amid those that underwent this protective surgical maneuver $(\mathrm{P}<0.001)$.

Disease-free survival at 4.5 years was $93 \%$ with protective vaginal closure and $74 \%$ in those without $(P<0.001)$. Minimally invasive surgery patients without protective vaginal closure had a 2.58-times higher risk of recurrence than the open approach $(\mathrm{HR}, 2.58 ; 95 \% \mathrm{Cl}$, 1.70 to $3.95 ; P<0.001)$. Moreover, those who underwent minimally invasive surgery with vaginal closure had similar rates of relapse to those who underwent open surgery $(\mathrm{HR}, 0.63 ; 95 \% \mathrm{Cl}, 0.15$ to 2.59 ; $\mathrm{P}<0.52$ ) (Figure 4B). These differences remained in patients with tumors $>2 \mathrm{~cm}$ (HR, 2.99; $95 \% \mathrm{Cl}, 1.78$ to $5.00 ; \mathrm{P}<0.001)$. but were not found in those with tumors $\leq 2 \mathrm{~cm}$ (HR, 1.96; $95 \% \mathrm{Cl}, 0.91$ to 4.27; $\mathrm{P}<0.09$ ).

Minimally invasive surgery patients without vaginal closure had 2.85-times risk of death when compared with those that underwent an open approach (HR, 2.85; 95\% Cl, 1.59 to $5.15 ; \mathrm{P}<0.001)$. We observed these differences in patients with tumors $>2 \mathrm{~cm}$ (HR, 2.71; $95 \% \mathrm{Cl}, 1.35$ to 5.46 ; $\mathrm{P}=0.005)$ and those with tumors $\leq 2 \mathrm{~cm}(\mathrm{HR}$, $3.33 ; 95 \% \mathrm{Cl}, 1.06$ to $10.46 ; \mathrm{P}=0.039$ ).

\section{DISCUSSION}

Our study showed that in a European population, minimally invasive surgery increases the risk of relapse and death among patients with early cervical cancer. There was a $10 \%$ difference at 4.5 -year diseasefree survival between surgical approaches, which is consistent with
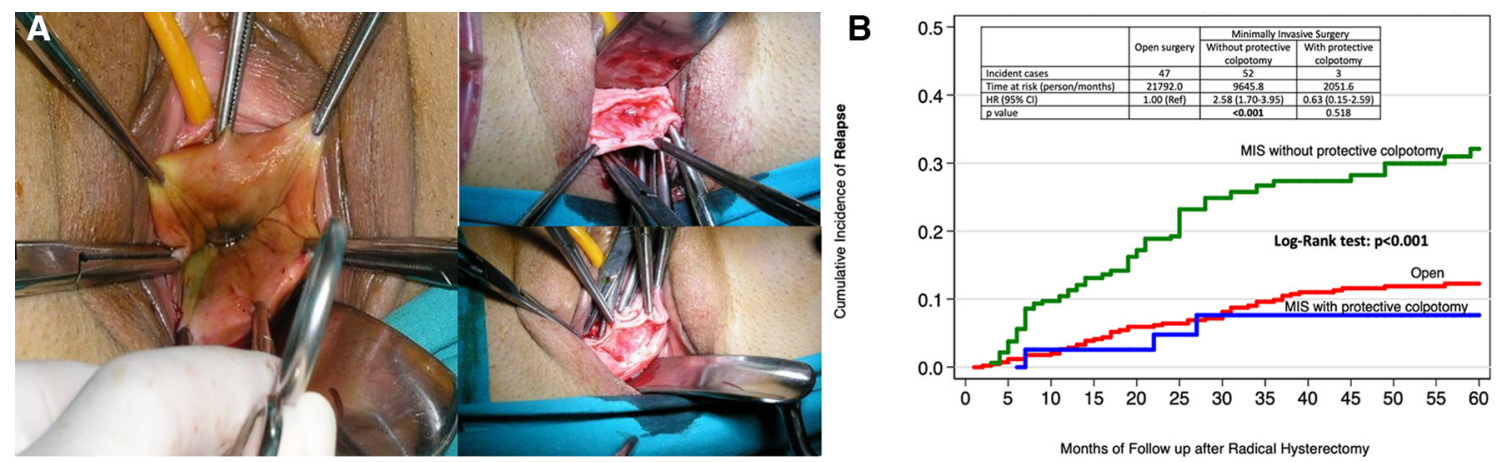

$$
\text { Months of Follow up after Radical Hysterectomy }
$$

Figure 4 (A) Protective maneuver for the closure of the vagina over the tumor at the time of colpotomy, either at the beginning or at the end of the laparoscopic procedure to avoid the spillage of the cervical tumor. Courtesy of Dr. Aureli Torne and Dr. Jaume Pahisa. Clinic Hospital Barcelona Spain. (B) Hazard ratios and 95\% confidence intervals for the risk of relapse by type of intervention in patients that underwent minimally invasive surgery (protective vaginal closure vs no protective vaginal closure) compared with patients with open radical hysterectomy adjusted using inverse probability weighting by propensity scores. 
the results of the LACC trial. This outcome particularly pertained to tumors $>2 \mathrm{~cm}$ in diameter. However, one should note that the absence of statistical significance in small tumors does not translate to an absence of effect, given the lower incidence of events in this low-risk group. In addition, in this study, avoiding the use of uterine manipulator and a protective vaginal closure to prevent tumor spillage leads to oncologic outcomes that are similar to those of open surgery.

As it pertains to the findings on the uterine manipulator in the minimally invasive procedure, it should be highlighted that although it technically facilitates the procedure, it increases the risk of relapse by 2.76 -times. Interestingly, patients that underwent a radical hysterectomy by minimally invasive surgery without the uterine manipulator had comparable outcomes to those with open surgery. This was noted in patients with tumors $>2 \mathrm{~cm}$ but not in those with tumors $\leq 2 \mathrm{~cm}$. In our study, patients that underwent minimally invasive surgery with protective vaginal closure showed similar rates of relapse to those who underwent open surgery.

Recently, two large nation-based retrospective studies, one from Denmark and the other from the Netherlands, failed to show differences in disease-free survival and overall survival comparing the open vs minimally invasive approach. ${ }^{27}{ }^{28}$ The Danish study compares two periods before and after the introduction of robotic surgery from 2005 to 2017. Compared with SUCCOR, the groups were metachronous with a significantly different median follow-up in both groups (113.0 vs 42.4 months). In that study, the percentage of different surgical approaches in each study group is not clearly defined and conization was not considered a potential confounding factor. The Dutch study evaluates the outcome in a weighted cohort with a propensity matching score. Unlike our study, it did not include adjuvant therapy, margin status, nor previous conization for the weighted cohort design.

Overall, the strengths of this study include a collaborative effort of 126 European institutions from 29 countries where comprehensive data was collected on 1272 patients. Our project gathers one of the most extensive groups of radical hysterectomies ever collected in Europe in patients with stage IB1 cervical cancer, who underwent surgery within 2 years. We designed a strict list of inclusion and exclusion criteria to minimize the implicit bias of a retrospective study. We required mandatory preoperative imaging and a list of fundamental pathological findings for inclusion. Also, we excluded all cases with previous cone biopsy. We believe that the best way of investigating the action of a specific surgical approach in early cervical cancer is in the untouched-entire tumor even though this is still a hypothesis. Another strength of our study is the use of an inverse probability of treatment weighting based on propensity score in an attempt to balance confounders between groups, such that selection bias for the surgical procedure was minimized. We recognize that our study still has not entirely answered why minimally invasive surgery was associated with worse survival in patients undergoing radical hysterectomy. However, findings from our study on uterine manipulator and protective maneuvers are hypothesis-generating and may offer some light to the etiology of the outcomes from the LACC trial.

SUCCOR is an observational retrospective study and thus we recognize a number of limitations. However, observational studies may contribute valuable evidence supporting causal associations when designed and conducted using rigorous methods. The flaws are dependent on outdated methodology, confounding factors, heterogeneity of reporting of results, lack of replication, and a failure to interpret findings within the limitations of observational research methodology ${ }^{29-31}$ Overall, the results reflect that in this large European population findings were consistent with recently published prospective data and confirmed by several retrospective population-based analyzes published since the LACC trial. ${ }^{8-15}$

There are two prospective randomized trials exploring the role of minimally invasive surgery in patients with cervical cancer. The first is the RACC trial, ${ }^{28}$ a Swedish multicentric prospective trial comparing robotic vs open surgery for the treatment of early-stage cervical cancer. The use of uterine manipulator is not allowed, and the closure of the vagina before colpotomy is recommended but not mandatory. The second one is a multicenter randomized controlled trial designed in China. ${ }^{32}$ Again, in their protocol, the use of uterine manipulator and the method of vaginal excision is to be reported.

In summary, our study showed worse disease-free survival in patients undergoing minimally invasive radical hysterectomy when compared with the open approach. We also found that avoiding the use of the uterine manipulator or closing the vagina over the tumor might hypothetically improve the results of radical hysterectomy by minimally invasive surgery. These findings should be confirmed with prospective data. However, it is questionable whether one can genuinely repeat a prospective randomized trial in this patient population based on increasing physician hesitation to offer minimally invasive surgery or patient willingness for randomization. We conclude that in the SUCCOR study, in a European population, minimally invasive surgery was associated with significantly worse oncologic outcomes than open surgery. Avoiding the uterine manipulator and a meticulous closure of the vagina over the tumor to prevent tumor spillage may improve the outcomes of minimally invasive surgery. We look forward to the results of the ongoing randomized trials.

\footnotetext{
Author affiliations

${ }^{1}$ Department of Obstetrics and Gynecology, Clinica Universidad de Navarra, Madrid, Spain

${ }^{2}$ Istituto Europeo di Oncologia, Milano, Italy

${ }^{3}$ Surgery, Institut Bergonie, Bordeaux, France

${ }^{4}$ Department of Preventive Medicine and Public Health, Universidad de Navarra,

Pamplona, Spain

${ }^{5}$ Principe de Asturias University Hospital, Alcala de Henares, Spain

${ }^{6}$ Emergency County Hospital, Targu Mures, Romania

${ }^{7}$ Policlinico A Gemelli, Roma, Italy

${ }^{8}$ Queen Elizabeth Hospital, Gateshead, UK

${ }^{9}$ Amsterdam University Medical Centre, Amsterdam, Netherlands

${ }^{10}$ University Oncologic Hospital, Sofia, Bulgaria

${ }^{11}$ National Center of Oncology, Baku, Azerbaijan

${ }^{12}$ Center for Advanced Endoscopic Gynecologic Surgery, Avellino, Italy

${ }^{13}$ Gynecology, Centre Oscar Lambret, Lille, France

${ }^{14}$ University Maternal Hospital Canary Islands, Las Palma, Spain

${ }^{15}$ Fondazione IRCCS Istituto Nazionale dei Tumori, Milano, Italy

${ }^{16}$ Saglik Bilimleri University Antalya Research and Training Hospital, Antalya, Turkey

${ }^{17}$ Department of Obstetrics and Gynecology, University of Prague, Prague, Czech

Republic

${ }^{18}$ Oncogynecology, Kazahskij Naucno-issledovatel'skij Institut Onkologii i Radiologii,

Almaty, Kazakhstan

${ }^{19}$ Department of Gynecologic Oncology, Zekai Tahir Burak Women's Health

Education and Research Hospital, Ankara, Turkey

${ }^{20}$ IPO-PORTO, Porto, Portugal

${ }^{21}$ LISOD - Israeli Oncological Hospital, Kyiv, Ukraine

${ }^{22}$ Azienda Ospedaliero-Universitaria di Bologna Policlinico SantOrsola-Malpighi,

Bologna, Italy

${ }^{23}$ Obstetrics and Gynecology; Unit of Gynecologic Oncology; Institute of Obstetrics and Gynecology; Faculty of Medicine, University of Debrecen, Debrecen, Hungary

${ }^{24}$ General Hospital of Thessaloniki Papageorgiou, Thessaloniki, Greece
} 


\section{Original research}

${ }^{25}$ Clinical Hospital Center, Zagreb, Croatia

${ }^{26}$ Oncological Gynecology, Lower Silesian Cancer Center, Wroclaw, Poland

${ }^{27}$ Gynecological Oncology, Radboudumc, Nijmegen, Netherlands

${ }^{28}$ Gynaecology, LUMC, Leiden, Netherlands

${ }^{29}$ Obstetrics and Gynecology, University of Liege, Liege, Belgium

${ }^{30}$ Division of Gynecologic Oncology, 1st Department of Obstetrics and Gynecology,

Alexandra Hospital, National and Kapodistrian University of Athens, Athens, Greece

${ }^{31}$ Clinical Hospital Center Rijeka, Rijeka, Croatia

${ }^{32}$ Department of Gynecology and Oncology, Jagiellonian University, Krakow, Poland

${ }^{33}$ Lviv Oncology Center, Lviv, Ukraine

${ }^{34}$ North-Western State Medical University. N.N. Petrov Research Institute of Oncology, St Petersburg, Russia

${ }^{35}$ Instituto Português de Oncologia de Lisboa, Lisboa, Portugal

${ }^{36}$ University Hospitals of Leicester NHS Trust, Leicester, UK

${ }^{37}$ Medical University of Graz, Graz, Steiermark, Austria

${ }^{38}$ Obstetrics and Gynecology, Tampere University Hospital, Tampere, Finland

${ }^{39}$ Oncogynecology, Grigorev Institute for Radiology, Kharkiv, Ukraine

${ }^{40}$ Institut Curie, Paris, île-de-France, France

${ }^{41}$ Department of Medicine and Surgery, University of Milan-Bicocca, Milano, Italy

${ }^{42}$ Clinic of Obstetrics and Gynecology, Hospital San Gerardo, Monza, Italy

${ }^{43}$ North Estonia Medical Centre, Tallinn, Estonia

${ }^{44}$ Bellvitge University Hospital, L'Hospitalet de Llobregat, Catalunya, Spain

${ }^{45}$ Obstetrics and Gynecology, Clinica Universidad de Navarra, Pamplona, Spain

${ }^{46}$ Obstetrics and Gynecology, Universidad de Navarra, Pamplona, Spain

${ }^{47}$ Obstetrics and Gynecology, School of Medicine; University of Navarra, Pamplona, Spain

\section{Presented at}

Plenary Session at the 21st European Congress on Gynaecological Oncology on November 2-5, 2019 in Athens, Greece.

Correction notice This article has been corrected since it was published Online First. The collaborator names Elif Aksahin, Fuat Demirkiran, Meelis Leht and Arno Uppin were written incorrectly and Özgür Akbayir was omitted from the list. This has been corrected.

Twitter Luis Chiva @\#lchiv4, Dilyara Kaidarova @r.bolatbekova@gmail.com, Rasiah Bharathan @RasiahBharathan and Juan Luis Alcazar @Juan_L_Alcazar

Acknowledgements We thank our colleagues, Dr. Matias Jurado, Dr. Antonio Gil and Dr. Antonio Gonzalez-Martin, for their invaluable support when designing this project. A special recognition to the Medical Editor of the International Journal of Gynecological Cancer for his continuous assistance in improving this Manuscript. We thank the ESGO Council for the diffusion of the SUCCOR study among our members

Collaborators On behalf of the SUCCOR study Group: Nabil Abdalla, Özgür Akbayir, Sedat Akgöl, Elif Aksahin, Shamistan Aliyev, Maria Alonso-Espias, Igor Aluloski, Claudia Andrade, Nikola Badzakov, Rosa Barrachina, Giorgio Bogani, Eduard-Aexandru Bonci, Hélène Bonsang-Kitzis, Felix Boria, Cosima Brucker, Laura Cárdenas, Andrea Casajuana, Pere Cavalle, Jorge Cea, Benito Chiofalo, Gloria Cordeiro, Pluvio Coronado, Maria Cuadra, Javier Díez, Teresa Diniz da Costa, Santiago Domingo, Lukas Dostalek, Fuat Demirkiran, Diego Erasun, Mathias Fehr, Sergi Fernandez-Gonzalez, Annamaria Ferrero, Soledad Fidalgo, Gabriel Fiol, Khadra Galaal, José García, Gerhard Gebauer, Fabio Ghezzi, Juan Gilabert, Nana Gomes, Elisabete Gonçalves, Virginia Gonzalez, Frederic Grandjean, Miriam Guijarro, Frédéric Guyon, Jolien Haesen, Gines HernandezCortes, Sofía Herrero, Imre Pete, Ioannis Kalogiannidis, Erbil Karaman, Andreas Kavallaris, Lukasz Klasa, Ioannis Kotsopoulos, Stefan Kovachev, Meelis Leht, Arantxa Lekuona, Mathieu Luyckx, Michael Mallmann, Gemma Mancebo, Aljosa Mandic, Nabil Manzour, Tiermes Marina, Victor Martin, María Belén MartínSalamanca, Alejandra Martinez, Gesine Meili, Gustavo Mendinhos, Liliana Mereu, Milena Mitrovic, Sara Morales, Enrique Moratalla, Bibiana Morillas, Eva Myriokefalitaki, Maja Pakižlmre, Stamatios Petousis, Laurentiu Pirtea, Natalia Povolotskaya, Sonia Prader, Alfonso Quesada, Mikuláš Redecha, Fernando Roldan, Philip Rolland, Reeli Saaron, Cosmin-Paul Sarac, Jens-Peter Scharf, Špela Smrkolj, Rita Sousa, Artem Stepanyan, Vladimír Študent, Carmen Tauste, Hans Trum, Taner Turan, Manuela Undurraga, Arno Uppin, Alicia Vázquez, Ignace Vergote, George Vorgias and Ignacio Zapardiel.

Contributors All authors contributed meaningfully to the conception or design of the work or the acquisition, analysis, or interpretation of data for the study. The authors confirm the completeness and accuracy of the data and analyses, the fidelity of the study to the protocol, and the final approval of the version to be published.

Funding The authors have not declared a specific grant for this research from any funding agency in the public, commercial, or not-for-profit sectors.

Competing interests None declared.

Patient consent for publication Not required.

Provenance and peer review Not commissioned; externally peer reviewed.

Data availability statement Data are available upon reasonable request. Data will be available upon the requirement of the reviewers at any time.

\section{ORCID iDs}

Luis Chiva http://orcid.org/0000-0002-1908-3251

Fabrice Narducci http://orcid.org/0000-0001-5809-3535

Mehmet Mutlu Meydanli http://orcid.org/0000-0001-6763-9720

Anna Myriam Perrone http://orcid.org/0000-0003-3140-4772

Marcin A Jedryka http://orcid.org/0000-0001-8935-0311

Vladyslav Sukhin http://orcid.org/0000-0002-4403-3707

Daniel Vázquez-Vicente http://orcid.org/0000-0002-9618-5606

Juan Luis Alcazar http://orcid.org/0000-0002-9700-0853

\section{REFERENCES}

1 Wertheim E. Zur frage der radicaloperation beim uteruskrebs. Arch. Gynak. 1900;61:627-68.

2 Querleu D. Radical hysterectomies by the Schauta-Amreich and Schauta-Stoeckel techniques assisted by celioscopy. J Gynecol Obstet Biol Reprod 1991;20:747-8.

3 Nezhat CR, Burrell MO, Nezhat FR, et al. Laparoscopic radical hysterectomy with paraaortic and pelvic node dissection. $\mathrm{Am} \mathrm{J}$ Obstet Gynecol 1992;166:864-5.

4 Zhao Y, Hang B, Xiong G-W, et al. Laparoscopic radical hysterectomy in early stage cervical cancer: a systematic review and meta-analysis. J Laparoendosc Adv Surg Tech A 2017;27:1132-44.

5 Park DA, Yun JE, Kim SW, et al. Surgical and clinical safety and effectiveness of robot-assisted laparoscopic hysterectomy compared to conventional laparoscopy and laparotomy for cervical cancer: a systematic review and meta-analysis. Eur J Surg Oncol 2017;43:994-1002.

6 Hong JH, Choi JS, Lee JH, et al. Can laparoscopic radical hysterectomy be a standard surgical modality in stage IA2-IIA cervical cancer? Gynecol Oncol 2012;127:102-6.

7 Ramirez PT, Soliman PT, Schmeler KM, et al. Laparoscopic and robotic techniques for radical hysterectomy in patients with earlystage cervical cancer. Gynecol Oncol 2008;110:S21-4.

8 Ramirez PT, Frumovitz M, Pareja R, et al. Minimally invasive versus abdominal radical hysterectomy for cervical cancer. N Engl J Med 2018;379:1895-904.

9 Melamed A, Margul DJ, Chen L, et al. Survival after minimally invasive radical hysterectomy for early-stage cervical cancer. $N$ Engl J Med 2018;379:1905-14.

10 Doo DW, Kirkland CT, Griswold LH, et al. Comparative outcomes between robotic and abdominal radical hysterectomy for IB1 cervical cancer: results from a single high volume institution. Gynecol Oncol 2019;153:242-7.

11 National cancer registration and analysis service (NCRAS) cervical cancer surgery analysis, 2019. Available: https://www.bgcs.org. uk/wp-content/uploads/2019/07/NCRAS-cervical-cancer-surgeryanalysis-May-2019-final.pdf [Accessed July 3rd 2020].

$12 \mathrm{Kim} \mathrm{SI}$, Lee M, Lee S, et al. Impact of laparoscopic radical hysterectomy on survival outcome in patients with FIGO stage IB cervical cancer: a matching study of two institutional hospitals in Korea. Gynecol Oncol 2019;155:75-82.

13 Cusimano MC, Baxter NN, Gien LT, et al. Impact of surgical approach on oncologic outcomes in women undergoing radical hysterectomy for cervical cancer. Am J Obstet Gynecol 2019;221:619.e1-619.e24.

14 Uppal S, Gehrig P, Vetter MH, et al. Recurrence rates in cervical cancer patients treated with abdominal versus minimally invasive radical hysterectomy: a multi-institutional analysis of 700 cases. $J$ Clin Oncol 2019;37:5504-5.

15 Chen X, Zhao N, Ye P, et al. Comparison of laparoscopic and open radical hysterectomy in cervical cancer patients with tumor size $\leq 2$ cm. Int J Gynecol Cancer 2020;30:564-71.

16 National Comprehensive Cancer Network. NCCN clinical practice guidelines in oncology cervical cancer, 2019. Available: https://www. 
nccn.org/professionals/physician_gls/pdf/cervical.pdf [Accessed July 3rd 2020.].

17 Querleu D, Cibula D, Concin N, et al. Laparoscopic radical hysterectomy: a European Society of Gynaecological Oncology (ESGO) statement. Int J Gynecol Cancer 2020;30.

18 ESMO Guidelines Committee. e-Update-cervical cancer treatment recommendations, 1 April 2020. authors: ESMO guidelines Committee. Available: https://www.esmo.org/guidelines/ gynaecological-cancers/cervical-cancer/eupdate-cervical-cancertreatment-recommendations [Accessed July 3rd 2020].

19 Chiva L, Cibula D, Querleu D. Minimally invasive or abdominal radical hysterectomy for cervical cancer. $N$ Engl J Med 2019;380:793:793-4.

20 Chiva L, Chacon E, Carriles I, et al. European perspective on surgical approach in early cervical cancer after LACC trial: an international ESGO survey. Int J Gynecol Cancer 2019;29:A20-1.

21 Krizova A, Clarke BA, Bernardini $M Q$, et al. Histologic artifacts in abdominal, vaginal, laparoscopic, and robotic hysterectomy specimens: a blinded, retrospective review. Am J Surg Pathol 2011;35:115-26.

22 Kanao H, Matsuo K, Aoki Y, et al. Feasibility and outcome of total laparoscopic radical hysterectomy with no-look no-touch technique for FIGO IB1 cervical cancer. J Gynecol Oncol 2019;30:e71.

23 Köhler C, Hertel H, Herrmann J, et al. Laparoscopic radical hysterectomy with transvaginal closure of vaginal cuff - a multicenter analysis. Int J Gynecol Cancer 2019;29:845-50.

24 Clinical Trials.gov. SUCCOR-Surgery in cervical cancer comparing different surgical aproaches in stage IB1 cervical cancer (SUCCOR)

25 Austin PC, Stuart EA. Moving towards best practice when using inverse probability of treatment weighting (IPTW) using the propensity score to estimate causal treatment effects in observational studies. Stat Med 2015;34:3661-79.
26 Twu N-F, Ou Y-C, Liao C-I, et al. Prognostic factors and adjuvant therapy on survival in early-stage cervical adenocarcinoma/ adenosquamous carcinoma after primary radical surgery: a Taiwanese Gynecologic Oncology Group (TGOG) study. Surg Oncol 2016;25:229-35

27 Wenzel HHB, Smolders RGV, Beltman JJ, et al. Survival of patients with early-stage cervical cancer after abdominal or laparoscopic radical hysterectomy: a nationwide cohort study and literature review. Eur J Cancer 2020;133:14-21.

28 Jensen PT, Schnack TH, Frøding LP, et al. Survival after a nationwide adoption of robotic minimally invasive surgery for earlystage cervical cancer - a population-based study. Eur J Cancer 2020;128:47-56.

29 Lederer DJ, Bell SC, Branson RD, et al. Control of confounding and reporting of results in causal inference studies. guidance for authors from editors of respiratory, sleep, and critical care journals. Ann Am Thorac Soc 2019;16:22-8.

30 Kitsios GD, Dahabreh IJ, Callahan S, et al. Can we trust observational studies using propensity scores in the critical care literature? A systematic comparison with randomized clinical trials. Crit Care Med 2015;43:1870-9.

31 Gershon AS, Jafarzadeh SR, Wilson KC, et al. Clinical knowledge from observational studies. everything you wanted to know but were afraid to ask. Am J Respir Crit Care Med 2018;198:859-67.

32 Falconer H, Palsdottir K, Stalberg K, et al. Robot-assisted approach to cervical cancer (RACC): an international multi-center, open-label randomized controlled trial. Int $J$ Gynecol Cancer 2019;29:1072-6.

33 Chao X, Li L, Wu M, et al. Efficacy of different surgical approaches in the clinical and survival outcomes of patients with early-stage cervical cancer: protocol of a phase III multicentre randomised controlled trial in China. BMJ Open 2019;9:e029055. 